\title{
Non Biologic Complex Drug Concept: Experiences with Iron Sucrose and Low Molecular Weight Heparin
}

\section{Jacques Rottembourg ${ }^{1^{*}}$ and Huub Schellekens ${ }^{2}$}

${ }^{1}$ Department of Nephrology, Groupe Hospitalier Pitié-Salpêtrière- Paris, France

${ }^{2}$ Department of Pharmaceutical Sciences, Utrecht University, Utrecht, Netherlands

"Corresponding author: Jacques Rottembourg, Department of Nephrology, Groupe Hospitalier Pitié-Salpêtrière, 83 Boulevard de l'Hôpital, 75013 Paris, France, Tel: 0033143590821; Fax: 0033143590824; E-mail: jacques.rottembourg@wanadoo.fr

Rec date: Jan 26, 2014, Acc date: Mar 06, 2014, Pub date: Mar 10, 2014

Copyright: (c) 2013 Rottembourg J, et al. This is an open-access article distributed under the terms of the Creative Commons Attribution License, which permits unrestricted use, distribution, and reproduction in any medium, provided the original author and source are credited.

\begin{abstract}
When the patent of a small molecule drug expires, generics may be introduced. They are considered therapeutically equivalent once pharmaceutical equivalence (i.e. identical active substances) and bioequivalence (i.e. comparable pharmacokinetics) have been established in cross-over volunteer study. However this generic paradigm cannot be applied to complex drugs as biologics. For copies of biologics EMA, and FDA, have introduced a new regulatory bio-similar pathway which mandate clinical trials to show therapeutic equivalence. However some complex drugs, such as iron-carbohydrate drugs, low molecular weight heparins (named Non Biologic Complex Drugs [NBCD]), regulatory guidance is still mostly lacking. In this paper we will discuss therapeutic experiences with these different classes of complex drugs and their specificity, to provide scientific arguments for consideration for a new regulatory framework.
\end{abstract}

Keywords: Generic; Biosimilars; Therapeutic equivalence; Ironcarbohydrate complexes; Low molecular weight Heparin

\section{The Non Biologic Complex Drug Concept}

When the patent of a classical small molecule drug expires, generics may be marketed if their therapeutic equivalence to the original drug has been established [1,2]. Conventional generics for an orally administered drug are considered to be therapeutically equivalent to a reference once pharmaceutical equivalence (i.e. identical active substances) and bioequivalence (i.e. comparable pharmacokinetics essentially on healthy volunteers) have been established in a cross over study and do not require formal clinical efficacy and safety studies. The therapeutic equivalence allows then the therapeutically interchangeability [3]. The acceptance intervals to show that bioequivalence for the logarithm transformed AUC and Cmax ratios lie within an acceptance range of $0.80-1.25$ for the $90 \%$ confidence intervals. In some cases the acceptance interval need to be tightened, and in other cases a wider acceptance may be acceptable.

The classical generic approach based on showing pharmaceutical equivalence and bioequivalence has been the basis of the introduction of many safe and effective alternatives to innovative medicines. However, this approach has been far only applied to products which can be fully characterized. For more complex molecules which are difficult to characterize such as proteins, the demonstration of bioequivalence requires an alternative approach.

Therapeutic proteins are the clinically most widely used class of drugs for which the classical generic paradigm cannot be used $[4,5]$. The molecular mass of most of the therapeutic proteins varies from 5 to $150 \mathrm{kDa}$, which is $25-1000$ times larger than the average small drug molecule. Nearly all therapeutic proteins are produced by living cells and these cells in general make mixtures of different proteins, e.g. by variation in the process of post-translation modification such as glycosylation. There are many tools to analyse the structure and in vitro activity of therapeutic proteins (biologics). However because of their complexity there is no combination of techniques that can completely describe all structural features of a protein drug. Currently, only limited data are available regarding the predictive value of pharmacokinetics studies for the clinical activities of biologics.

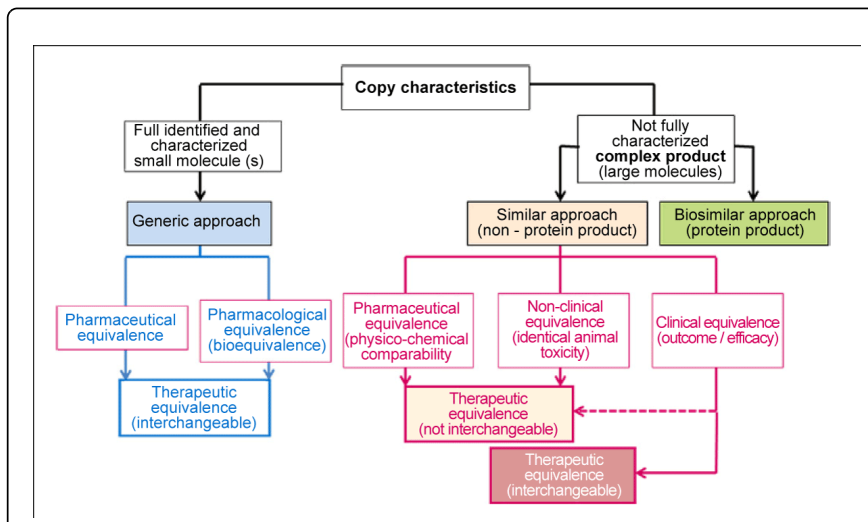

Figure 1: Algorithm for authorizations of copies (therapeutic equivalence): comparability of complex products by the similar pathway (issued from Schellekens $\mathrm{H}$, et al. [9]).

The most important difference between biologics and low molecular weight drugs is their immunogenicity [6]. Nearly all therapeutics proteins induce antibodies, irrespective of whether these proteins are partly non-human or completely human homologs. Because with proteins it is impossible to show two products to be identical, the term "bio-similar" was introduced in the European Union, which in 2004 adopted legislation to establish a comprehensive regulatory pathway for bringing bio-similar to the market $[7,8]$. Thus, 
bio-similars are defined as biological products which are similar, but not identical to reference products. They are submitted for separate marketing approval following patent expiration. To be allowed on the market the bio-similar product should be shown to be similar to the reference product in terms of quality, safety and efficacy. And in contrast with the regulatory pathway for generics of small molecules, this includes the need for comparative clinical data. Based on advice by the EMA/CHMP, the European commission has approved bio-similar versions of recombinant human EPO (rHuEPO), filgrastim and growth hormone.

Such pathway still does not exist for non-protein complex drugs. It is difficult to define a regulatory pathway which covers all possible complex drugs. However a case-by-case approach is not also satisfactory from a regulatory point of view. In our view the generic approach should be limited to products which can be fully characterized and of which the physical-chemical characteristics predict completely the biological effects and pharmacokinetic data can be used as surrogate for clinical efficacy. For all other products the biosimilar-like approach should be applied, which is mostly established for proteins, but not for non-protein large or multisource products (Figure 1). This approach could be modified depending on a number of factors: the availability of validated pharmacodynamics biomarkers, extrapolated indications, animal studies, and clinical studies to show interchangeability. It may concern drugs such as Low Molecular Weight Heparins (LMWH) (considered as bio-similar by the European Medicine Agency, but as small molecule drug by FDA) liposomal drugs, glatiramoids, and iron-carbohydrate complexes [9].

\section{Complex Iron-Carbohydrate Drugs (Iron Sucrose-Iron Sucrose Similar)}

Iron-Sucrose (IS) is the main representative of the ironoxyhydroxide carbohydrate drugs, a class of colloidal IV iron preparations. It is used for the treatment of iron deficiency anaemia associated with chronic kidney disease, pregnancy, malabsorption, and blood donation [10-12]. IS has a favourable safety profile when administered IV as outlined in the European Best Practice Guidelines [13]. IS are complex macromolecules, and their physico-chemical and biological properties are closely dependent on the manufacturing process such that subtle structural modifications may affect the stability of the preparation $[9,14]$. The characteristics of iron compounds used in IV preparations depend in particular on the interaction between the iron (III)-hydroxyde core and the surrounding carbohydrates. Stability is of paramount importance since weakly bound iron may dissociate too rapidly and catalyse the generation of reactive oxygen species [15] that in turn induce oxidative stress and inflammation. Potentially, an unstable iron complex could have safety and efficacy implications, especially in chronically ill individual such as Haemodialysis (HD) patients in whom dialysis and comorbidities already confer an increased burden of oxidative stress and inflammation $[16,17]$.

Several Iron Sucrose Similar (ISS) preparations have been introduced for the treatment of iron deficiency anaemia worldwide [18] on the basis that they can be considered therapeutically equivalent to the originator IS. However recent analytical tests and studies in rat models have demonstrated differences between ISS preparations and the originator IS in term of physico-chemical structure and their effect on inflammatory, hemodynamic and functional markers $[14,19]$.
In the dialysis unit in Paris, IS was used to correct iron deficiency anaemia in HD patients for 6 years prior to June 2009 at which point, the unit switched to the ISS for economic reasons. An observational retrospective and prospective analysis was undertaken to evaluate the impact of the switch from the originator IS to the ISS on Haemoglobin $\mathrm{Hb})$ levels, iron parameters and dosing requirements for IV iron and Erythropoiesis-Stimulating Agents (ESA) [20]. The impact was assessed in a sequential observational study comparing two periods of 27 weeks each in 75 stable HD patients receiving IV iron weekly and ESA once every 2 weeks. Patients received IS in the first period (P1) and ISS in the second period (P2).

Mean $\mathrm{Hb}$ value was $11.78 \pm 0.99 \mathrm{~g} / \mathrm{dl}$ during $\mathrm{P} 1$ and $11.48 \pm 0.98$ $\mathrm{g} / \mathrm{dl}$ during P2 (p-value $=0.01$ ). Mean serum ferritin was similar for both treatment periods $(\mathrm{P} 1,534 \pm 328 \mu \mathrm{g} / \mathrm{l} ; \mathrm{P} 2495 \pm 280 \mu \mathrm{G} / \mathrm{l}, \mathrm{p}=0.25)$ but mean TSAT during P1 $(49.3 \pm 10.9 \%)$ was significantly higher than during P2 $(24.5 \pm 9.4 \%, \mathrm{p}<0.0001)$. The mean dose of IV iron per patient per week was $45.58 \pm 32.55 \mathrm{mg}$ in $\mathrm{P} 1$ and $61.36 \pm 30.98 \mathrm{mg}$ in $\mathrm{P} 2(+34.6 \%)$, while the mean ESA dose was $0.58 \pm 0.52$ and $0.66 \pm 0.64$ $\mu \mathrm{g} / \mathrm{kg} /$ week respectively $(+13.8 \%)$. Total mean anaemia drug cost increased in P2 by $11.9 \%$ compared to P1. The mean fortnightly IV iron dose and concomitant ESA dose throughout the study versus achieved $\mathrm{Hb}$ levels are shown in Figure 2.

In this population of stable HD patients, switch to an ISS was associated with a significant reduction in $\mathrm{Hb}$ level and reduced iron indices. This deterioration was observed despite an increase in both IV iron and ESA dosing. Variations in complex structure and stability of ISS are likely to have accounted for the differences in $\mathrm{Hb}$ control, since the kinetics of iron dissociation influence the pattern of iron release, distribution and storage [15]. In this population of iron-deficient individuals, TSAT values decreased dramatically after the switch, indicating that less iron was available for erythropoiesis. This may signify that iron released from the ISS had been sequestered by other compartments of the body such as the liver, consistent with the more extensive iron deposits observed in liver tissues within the ISS groups of experimental studies [14,19]. Differences in efficacy and safety between originator and similar preparations have been more recently published [21-23]. Both the FDA and EMA acknowledge that iron preparations cannot be authorized by the so far well-established generic approval paradigm for small molecules which is based on the sameness of the product shown by physico-chemical full characterization and a bioequivalence in healthy volunteers [24,25]. The need to also characterize the properties by qualitative and quantitative in vitro testing is recognized in the FDA draft guidance on IV iron carbohydrate complexes [25]. In contrast the EMA sets priority on a nonclinical equivalence testing approach, including the comparative in vivo assessment of cellular damage by labile iron [24]. Recently the FDA has solicited a contractor for a prospective 3-year study combining both approaches from FDA and EMA in the evaluation of Nulecit ${ }^{\mathrm{TM}}$ with Ferlecit ${ }^{\mathrm{TM}}$ [26]. 


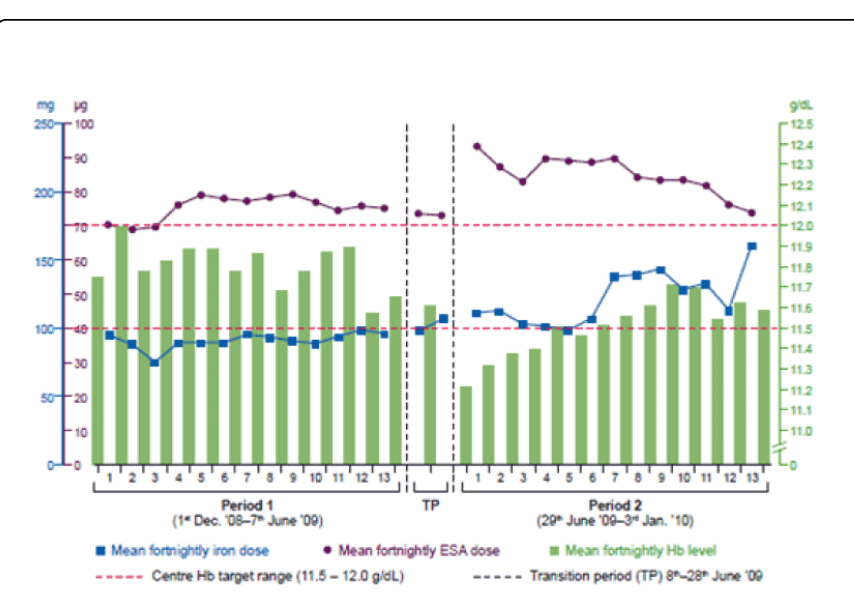

Figure 2: Mean fortnightly dose of IV iron (mg) and ESA (Darbepoetin- $\alpha[\mu \mathrm{g}])$ versus achieved $\mathrm{Hb}$ levels $(\mathrm{g} / \mathrm{dl})$. Based on the study by Rottembourg et al. [20]

\section{Low Molecular Weight Heparins (LMWH)}

The complexity of LMWHs is given from the starting material. UFH (Unfractionated Heparin) is an incompletely characterized heteropolymer of 48 theoretical disaccharide variants (building blocks), extracted and purified from animal mucosa. LMWHs are obtained through specific and proprietary depolymerisation processes of UFH with each process resulting in a distinct end product. LMWHs are comprised of a mixture of thousands of oligosaccharides (complex sugars) also incompletely characterized and which may differ between copies.

Each product displays a large spectrum of anticoagulant and nonanticoagulant properties. Structure-activity relationships have not been fully established due to an inability to separate the entire oligosaccharide mixture into individual and fully characterized oligosaccharides.

In Europe, in June 2006, as per the CHMP Biosimilar Working Party (BWP) recommendation, the Coordination Group for Mutual recognition and Decentralized procedures-human (CMDh) identified Low Molecular Weight Heparins as biological medicinal products. The CHMP released a specific guideline in March 2009 addressing the non-clinical and clinical aspects of the development of similar biological medicinal products containing LMWHs [27].

In contrast, the US FDA currently regulates LMWHs and other product classes such as insulin or human growth hormones as drugs and not as biologics, mainly for historical reasons. Nevertheless, the FDA has recognized that LMWHs are not fully characterized and that the classical criteria applied to standard generic drugs are inadequate for these products. Recently the FDA approved a generic LMWH and described the evaluation criteria that were considered for this decision [28]. FDA has yet to rule on several other abridged applications for generic LMWHs pending since 2003.

The worldwide heparin demand for 2009 is estimated to be 800 million doses requiring 1.2 billion pig gut mucosa, a figure that approaches the total worldwide pig production. Therefore, the complete pharmaceutical control of the sourcing is mandatory as the bulk product stability and process performance requires a first heparin extraction step is done locally in large scale prearranged facilities. This was evident from the 2008 heparin contamination case from China raw material with OSCS (Over Sulfated Chondroitin Sulfate) resulting in a significant number of fatal cases and resolved by an updated pharmacopoeia monographs. LMWHs manufacturers must guarantee an exclusive use of controlled sources and quality assurance resulting in a full traceability from the slaughterhouse to the finished product.

There is currently no enabling technology to completely characterize LMWHs and to conduct a full pharmaceutical comparability (equivalence) exercise. Even if specific differences in the PK and PD profile between UFH and LMWH exist, no classical PK profile may be determined for LMWHs due to the multi-component composition (heterogeneity) and (a) PD (pharmacodynamics) biomarker(s) as surrogate(s) is needed. The inhibition of activated Stuart factor (anti-factor Xa activity) and the inhibition of thrombin activation reflect the main anticoagulant activities of LMWH, but these correlate poorly with clinical outcomes and are not predictive of the PK profile of each of the active ingredients present in the mixture.

As a first part of the therapeutic (and safety) comparability exercise in humans, CHMP Guidelines on biosimilar LMWHs recommend for $\mathrm{PK} / \mathrm{PD}$ comparison a randomized single dose two way crossover study using SC (and depending on the case, IV) administration in healthy volunteers measuring a variety of biomarkers (anti-Xa, anti-IIa, TFPI, etc.). We recommend investigating both low and high dosages to reflect the approved clinical use and to take into account the nonlinearity of the dose-response curves. Patients with severe renal impairment should also be considered in this evaluation in order to detect possible differences not detected in healthy volunteers. An acceptable range for such a comparability exercise would be similar to that for bioequivalence ( $80-125 \%$ of the innovator product).

Heparin products, including LMWHs, can elicit immunogenicity reactions like the Heparin Induced Thrombocytopenia (HIT). The incidence of developing HIT antibodies is estimated up to $8 \%$ of heparinized patients and less than $1 \%$ for those exposed to LMWH [29]. Approximately $1-5 \%$ of patients on heparin will progress to develop HIT with thrombocytopenia, at least one-third suffering from thrombosis [29]. Considering the vast number of patients exposed to heparins, HIT is a common drug-induced immunologic response. CHMP recommends following the Immunogenicity Guidance (CHMP/14327/06) for LMWHs. In addition to that guidance, we recommend that the assays for HIT antibodies be carefully standardized based on serum from patients with HIT.

The criteria recently described by the FDA for approving a generic LMWH do not include a head-to-head clinical trial vs the reference product [28]. In contrast therapeutic equivalence (clinical efficacy) according to CHMP should be demonstrated through at least one adequately powered, randomized, double-blind, parallel group clinical trials. Because of the high prevalence of Venous Thrombo-Embolism (VTE), a trial should be preferably conducted in patients undergoing major (orthopedic) surgery and should follow a strict equivalence design. The CHMP request for such trial on top of a thorough comparability exercise, probably results from the fact that LMWHs are not fully characterized and that their structure-effect relationship is not fully elucidated. The clinical trial is aimed at assessing the clinical relevance of the differences both detected and undetected during the comparability exercise. Following demonstration of therapeutic equivalence in one indication in surgical patients at high risk of VTE, CHMP guidelines indicate that extrapolation to all other indications may be acceptable if appropriately justified. It must be stressed that 
LMWH for prevention of VTE, but also for treatment of venous and arterial thrombosis with different dose regimens and/or routes of administration due also to major differences in the pathophysiology of venous and arterial thrombosis. Venous thrombosis mainly related to clot formation, whereas platelet adhesion/aggregation and inflammatory response are critical to arterial thrombus formation [30]. Some heparins exhibit anti-inflammatory activity involving interactions with pathways independent of AT like P-selectin, proteins of the complement system, and the contact-kinin system [31,32]. Recent discoveries suggest that some oligosaccharides considered as "passive" ingredients for VTE prevention because they do not contain the classical AT binding site, exhibit pro-fibrinolytic activity and therefore would become "active" ingredients in presence of an existing clot (VTE or Acute Coronary Syndrome treatment). Consequently, justification of any extrapolation between the clinical outcomes in VTE prevention and clot treatment, as well as between the venous and arterial settings remains a complex issue. Therefore, biosimilarity between two LMWHs should be probably established rather in separate studies for prevention of VTE and for treatment in an arterial indication.

LMWHs have its own active ingredient structure and demonstrate a unique clinical profile as a result of their manufacturing process. As a consequence regulatory bodies such as FDA, as well as medical societies consider LMWHs as non-interchangeable [28,33]. The FDA has determined that the recently approved generic LMWH can be substituted for its reference product [28]. An update was made about the safety and bioequivalence of bio-similars with a focus on enoxaparin [34]. First side effects were recently published with generic enoxaparin [35].

Within the different CHMP guidance's there is no specific recommendation regarding substitution and interchangeability of biosimilar products. In a Q\&A document issued by the EMA it is stated that "since bio-similar and biological reference medicines are similar but not identical, the decision to treat a patient with a reference or a bio-similar medicine should be taken following the opinion of a qualified healthcare professional" [7]. However, ultimately substitution rules are under the direct supervision of the National Health Authorities of the member states.

\section{Conclusion}

An increasing number of NBCDs including nano-medicines become target for development and introduction of follow-on products. Recent clinical data with NBCD follow-on products (ironsucrose) approved everywhere as generics, revealed significant differences in efficacy and tolerability compared to the originator product. Moreover the differences observed in the attitude of different regulators faced to LMWH such as enoxaparin and the first side effects published with a generic, enhanced the fact that LMWH are biologic products. Accordingly, regulators are prompted to establish a global regulatory framework that considers the structural complexity and specific biological properties of NBCD and provides clear guidance for the development and documentation of safe and effective follow-on products. The experts from academia, industry, regulatory bodies, and knowledge institutes at the FIP 2012 workshop suggest a stepwise similarity approach that includes appropriate clinical and/or nonclinical studies that evaluate markers of PK PD and safety/efficacy in relevant patient populations. As long as proof of therapeutic equivalence and safety profiles by appropriate studies is missing, interchangeability and automatic substitution between NBCDs and there follow-on products should be discouraged. Overall, two critical reviews, of the current and emerging regulation of $\mathrm{NBCDs}$ and $\mathrm{NBCD}$ follow-on products, encourage further multidisciplinary research and consensus discussions among all stakeholders to develop guidance towards the definition of an NBCD and he development of NBCD follow-on products $[36,37]$.

\section{References}

1. Meredith PA (1996) Generic drugs. Therapeutic equivalence. Drug Saf 15: 233-242.

2. Chen ML, Shah V, Patnaik R, Adams W, Hussain A, et al. (2001) Bioavailability and bioequivalence: an FDA regulatory overview. Pharm Res 18: 1645-1650.

3. Al-Jazairi AS, Bhareth S, Eqtefan IS, Al-Suwayeh SA (2008) Brand and generic medications: are they interchangeable? Ann Saudi Med 28: 33-41.

4. Crommelin DJ, Bermejo T, Bissig M, Damiaans J, Kramer I, et al. (2005) Pharmaceutical evaluation of biosimilars: important differences from generic low-molecular weight pharmaceuticals. Eur J Hosp Pharm 1: 11-17.

5. Schellekens H (2005) Follow-on biologics: challenges of the "next generation". Nephrol Dial Transplant 20 Suppl 4: iv31-36.

6. Baumann A (2009) Nonclinical development of biopharmaceuticals. Drug Discov Today 14: 1112-1122.

7. European Medicines Agency (2008) Questions and answers on Biosimilars Medicines.

8. Moors E, Schellekens H (2010) The strengths and weaknesses of the European biosimilar regulatory pathway. Nat Biotechnol 28: 28-32.

9. Schellekens H1, Klinger E, Mühlebach S, Brin JF, Storm G, et al. (2011) The therapeutic equivalence of complex drugs. Regul Toxicol Pharmacol 59: 176-183.

10. Locatelli F, Aljama P, Bárány P, Canaud B, Carrera F, et al. (2004) Revised European best practice guidelines for the management of anaemia in patients with chronic renal failure. Nephrol Dial Transplant 19 Suppl 2: ii1-47.

11. National Collaborating Centre for Chronic Conditions (2006) Anaemia management in chronic kidney disease: national clinical guideline for management in adults and children. London: Royal College of Physicians, London, UK.

12. National Kidney Foundation-Kidney Disease Outcomes Quality Initiative (NKF-KDOQI) (2007) KDOQI clinical practice guideline and clinical practice recommendations for anemia in chronic kidney disease: 2007 update of hemoglobin target. Am J Kidney Dis 50: 471-530.

13. Bailie GR, Clark JA, Lane CE, Lane PL (2005) Hypersensitivity reactions and deaths associated with intravenous iron preparations. Nephrol Dial Transplant 20: 1443-1449.

14. Toblli JE, Cao G, Oliveri L, Angerosa M (2009) Differences between the original iron sucrose complex Venofer ${ }^{\circ}$ and the iron sucrose similar Generis $^{\circ}$, and potential implications. Port J Nephrol Hypert 23: 53-63.

15. Geisser P, Baer M, Schaub E (1992) Structure/histotoxicity relationship of parenteral iron preparations. Arzneimittelforschung 42: 1439-1452.

16. Piga A, Roggero S, Longo F, Ernst O, Rose C (2009) Evaluation and treatment of secondary iron overload. Disorders of erythropoiesis, erythrocytes and iron metabolism - European Society of Haematology Handbook: Club du Globule et du Fer, Marseille, France.

17. Goldberg LA (2010) Iron sucrose similars. Hospital Pharmacy Europe 48: 21-22.

18. Garcia-Fernandez N, Echeverria A, Sanchez-Ibarrola A, Páramo JA, Coma-Canella I (2010) Randomized clinical trial on acute effects of i.v. iron sucrose during haemodialysis. Nephrology (Carlton) 15: 178-183.

19. Toblli JE, Cao G, Oliveri L, Angerosa M (2009) Differences between original intravenous iron sucrose and iron sucrose similar preparations. Arzneimittelforschung 59: 176-190. 
Citation: Jacques Rottembourg and Huub Schellekens (2014) Non Biologic Complex Drug Concept: Experiences with Iron Sucrose and Low Molecular Weight Heparin. J Blood Lymph 4: 123. doi:10.4172/2165-7831.1000123

Page 5 of 5

20. Rottembourg J, Kadri A, Leonard E, Dansaert A, Lafuma A (2011) Do two intravenous iron sucrose preparations have the same efficacy? Nephrol Dial Transplant 26: 3262-3267.

21. Stein J, Dignass A, Chow KU (2012) Clinical case reports raise doubts about the therapeutic equivalence of an iron sucrose similar preparation compared with iron sucrose originator. Curr Med Res Opin 28: 241-243.

22. Martin-Malo A, Merino A, Carracedo J, Alvarez-Lara MA, Ojeda R, et al (2012) Effects of intravenous iron on mononuclear cells during the haemodialysis session. Nephrol Dial Transplant 27: 2465-2471.

23. Lee ES, Park BR, Kim JS, Choi GY, Lee JJ, et al. (2013) Comparison of adverse event profile of intravenous iron sucrose and iron sucrose similar in postpartum and gynecologic operative patients. Curr Med Res Opin 29: 141-147.

24. EMA committee for Medicinal Products for human Use (CHMP) (2011) Reflection paper on non-clinical studies for generic nanoparticle iron medicinal product applications. EMA/CHMP/SWP1000094/2011.

25. FDA (2012) Draft guidance on iron sucrose bioequivalence.

26. FDA. Therapeutic equivalence of generic iron complex products (solicitation for a stud on $\mathrm{Na}$ ferric gluconate, Nulecit (follow-on upon 505j approval) vs Ferrlecit (originator).

27. European Medicines Agency (2009) Guidelines on Non-clinical and clinical development of similar biological medicinal products containing low-molecular weight heparins.

28. US Food and Drug Administration (2009).

29. Ranchini M (2005) Heparin-induced thrombocytopenia: an update Thrombosis J 3: 14-21.
30. Buffon A, Biasucci LM, Liuzzo G, D'Onofrio G, Crea F, et al. (2002) Widespread coronary inflammation in unstable angina. N Engl J Med 347: 5-12.

31. Hostettler N, Naggi A, Torri G, Ishai-Michaeli R, Casu B, et al. (2007) Pselectin- and heparanase-dependent antimetastatic activity of nonanticoagulant heparins. FASEB J 21: 3562-3572.

32. Ludwig RJ, Alban S, Boehncke WH (2006) Structural requirements of heparin and related molecules to exert a multitude of anti-inflammatory activities. Mini Rev Med Chem 6: 1009-1023.

33. Ryan TJ, Antman EM, Brooks NH, Califf RM, Hillis L, et al. (1999) ACC/AHA guidelines for the management of patients with acute myocardial infarction J Am Coll Cardiol 34 : 890-911.

34. Jeske W, Walenga JM, Hoppensteadt D, Fareed J (2013) Update on the safety and bioequivalence of biosimilars - focus on enoxaparin. Drug Healthc Patient Saf 5: 133-141.

35. Gucalp A, Parameswaran R, Lacouture M, Abou-Alfa G, Soff G (2013) Skin necrosis induced by generic enoxaparin. Am J Hematol 88: 339.

36. Schellekens H1, Stegemann S, Weinstein V, de Vlieger JS, Flühmann B, et al. (2014) How to regulate nonbiological complex drugs (NBCD) and their follow-on versions: points to consider. AAPS J 16: 15-21.

37. Schellekens H, Stegemann S, Weinstein V, de Vlieger JS, Flühmann B, et al. (2014) How to regulate nonbiological complex drugs (NBCD) and their follow-on versions: points to consider. AAPS J 16: 15-21. 